\title{
Predictive Factors of Chemotherapy Initiation after Biliary Drainage for Advanced Biliary Tract Cancer: A Retrospective Multicenter Study
}

\author{
Miloud Azarfane ${ }^{1}$, Astrid Lièvre ${ }^{2}$, Hélène Senellart ${ }^{3}$, Brigitte Dessomme ${ }^{4}$, Pauline Guillouche ${ }^{5}$, Jérémy Meyer ${ }^{6}$, Jaafar \\ Bennouna $^{1}$, Thimothée Wallenhorst ${ }^{2}$, Maëva Salimon ${ }^{1}$, Jérôme Gournay ${ }^{1}$, Tamara Matysiak-Budnik ${ }^{1}$, Annie Lim ${ }^{7}, J^{\prime}$ Tien \\ Edeline $^{8}$, Yann Touchefeu ${ }^{1}$
}

\author{
1) IMAD, Department \\ of Gastroenterology and \\ Digestive Oncology, University \\ Hospital, Nantes; \\ 2) Department of \\ Gastroenterology, CHU \\ Pontchaillou, Rennes 1 \\ University; INSERM U1242, \\ Oncogenesis, Stress and \\ Signaling, Rennes; \\ 3) Integrated Center for \\ Oncology, Centre René \\ Gauducheau, Saint Herblain; \\ 4) Department of Public \\ Health, University Hospital, \\ Nantes; \\ 5) Department of \\ Gastroenterology, Clinique \\ Jules Verne, Nantes; \\ 6) Department of Radiology, \\ University Hospital, Nantes; \\ 7) Department of \\ Gastroenterology, Clinique \\ Santé Atlantique, Saint \\ Herblain; \\ 8) Centre Eugène Marquis, \\ Oncology Department, \\ Rennes, France
}

\author{
Address for correspondence: \\ Yann Touchefeu, MD, PhD \\ IMAD, Hépato- \\ Gastroentérologie \& \\ Oncologie Digestive \\ Hôtel Dieu, CHU de Nantes \\ 1, Place Alexis Ricordeau \\ 44000 Nantes, France \\ yann.touchefeu@chu-nantes.fr
}

Received: 12.10 .2020

Accepted: 25.03 .2021

\section{ABSTRACT}

Background \& Aims: In unresectable biliary tract cancers, the management of biliary obstruction is often the first step before introduction of chemotherapy. Our aim was to study the predictive factors of chemotherapy initiation after biliary drainage in a series of patients presenting with advanced biliary tract cancer and obstructive jaundice.

Methods: Data of all patients treated for unresectable biliary tract cancer with initial biliary obstruction requiring a drainage in six institutions, from January 2009 to January 2019, were retrospectively collected. Results: Among 82 patients included in this study (median age 68 years, men 61\%), 48 (59\%) received chemotherapy. Median overall survival was 4.9 months (0.2-38.7) in the group of patients who did not receive chemotherapy and 12.2 months (1.9-61.0) in chemotherapy group ( $\mathrm{HR}=2.93 ; 95 \% \mathrm{CI}: 1.6-5.3 ; \mathrm{p}<0.0001)$. In univariate analysis, younger age, male gender, Eastern Cooperative Oncology Group (ECOG) score $\leq 2$, high albumin level, low C-reactive protein level, and endoscopic drainage were significantly associated with introduction of chemotherapy. In multivariate analysis, only ECOG score $\leq 2$ at diagnosis ( $\mathrm{HR}=70.4 ; 95 \% \mathrm{CI}$ : 4.6-1097.6; $\mathrm{p}=0.002)$ and male gender $(\mathrm{HR}=5$; 95\%CI: 1.5-16.5; $\mathrm{p}=0.009)$, were significant independent predictive factors of chemotherapy introduction. Age and bilirubin level at diagnosis were not significant factors in multivariate analysis.

Conclusions: ECOG score $\leq 2$ and male gender were the only independent predictive factors of chemotherapy introduction in unresectable biliary tract cancers. Age or initial bilirubin level were not predictors for chemotherapy introduction. These results might help defining the initial therapeutic strategy.

Key words: biliary tract cancer - predictive factors - prognostic factors - jaundice.

Abbreviations: BTC: biliary tract cancer; CAR: C reactive protein /albumin ratio; CRP: C reactive protein; ECOG: Eastern Cooperative Oncology Group; OS: overall survival; PFS: progression free survival; ULN: upper limit normal.

\section{INTRODUCTION}

Biliary tract cancers (BTC) account for about $3 \%$ of all gastrointestinal tumors and are the second most common primary hepatic tumors. Biliary tract cancers include intrahepatic, peri-hilar, distal and gallbladder carcinomas. Most patients present with advanced and unresectable disease at diagnosis. In patients eligible for chemotherapy, a combination with cisplatin and gemcitabine remains the standard first line treatment [1]. Jaundice is present in the majority of cases at initial diagnosis, most commonly due to biliary tract obstruction and is associated with a poor prognosis [2-4]. Biliary drainage can be achieved by percutaneous transhepatic or by endoscopic methods. The drainage of the bile ducts aims to improve the symptoms related to cholestasis, such as jaundice, pruritus and pain, to reduce the risk of cholangitis, but also to improve the nutritional and general status of the patient. In advanced BTC with obstructive jaundice, biliary drainage is the first step of management. Chemotherapy is commonly discussed later after the patient has been referred to an oncologist. Some patients who are initially unfit for chemotherapy can have the clinical benefits of the biliary drainage and become secondarily eligible. The identification of predictive factors of initiation of chemotherapy could help to better define which patients are likely to receive chemotherapy and in whom the work-up 
(port implantation, referral to an oncologist, complete imaging, optimal nutritional management) should be anticipated early after the biliary drainage.

The aim of this study was to investigate the predictive factors of chemotherapy initiation in a series of patients presenting with advanced BTC and obstructive jaundice requiring biliary drainage.

\section{METHODS}

From January 2009 to January 2019, all patients with unresectable cholangiocarcinoma complicated by biliary obstruction requiring percutaneous or endoscopic drainage in six participating centers, were identified.

Inclusion criteria were age 18 or older, cytological or histological diagnosis of BTC, advanced unresectable disease, biliary obstruction managed by percutaneous or endoscopic drainage. The pathological specimen was obtained during the drainage by biliary brushing or by percutaneous biopsy at a different time. Clinical, biological and histological characteristics were collected using electronic medical records.

Chemotherapy was considered as performed if patients received at least one complete cycle of the chemotherapy regimen. Because all the patients had an initial imaging demonstrating bile duct dilation, this imaging was considered as the time of initial diagnosis. Overall survival (OS) was defined as the time between the diagnosis and death (from all causes). Progression free survival (PFS) was defined as the time from the first day of chemotherapy to disease progression or death from any cause, whichever occurred first. Data were last updated in June 2019.

Several potential predictive factors for initiation of chemotherapy were investigated: age, gender, Eastern Cooperative Oncology Group (ECOG) score, weight loss, localization of the tumor, cholangitis, pruritus, serum bilirubin level at diagnosis and at drainage time, albumin serum level, $\mathrm{C}$ reactive protein level (CRP), CRP/albumin ratio (CAR), leukocyte and neutrophil counts at diagnosis, time between diagnosis and drainage, drainage technique, early complication of drainage, failure of previous drainage procedures. Infection, hemorrhage or pancreatitis that occurred after the biliary drainage during the time of hospitalization were considered as early complications of biliary drainage, but the relation to the drainage procedure could not be accurately determined in this retrospective design.

The group of patients who received chemotherapy and the group of patients who did not receive chemotherapy after the drainage were compared. Clinical and demographic data, as well as laboratory tests values, were analyzed in univariate analysis. Comparisons were performed using the non-parametric Mann-Whitney test for continuous data and the Chi2 and Fisher test for categorial data. Variables with a p-value $<0.05$ or clinically relevant with a $\mathrm{p}<0.20$ in univariate analysis were included in the multivariate analysis, performed with the Cox proportional hazard model, with a significance level of $\mathrm{p}<0.05$. Kaplan-Meier survival curves for OS were generated, and these curves were compared using log-rank tests. PFS was analyzed with the log-rank test. Analyses were performed using the software Graph Pad Prism 6 and XLStat 2017. Results are expressed as median (min-max). A p value $<0.05$ was considered statistically significant.

It was a retrospective study including patients managed with standard care only. The majority of patient were dead or lost to follow-up at the time of data collection. The study was performed according to the Declaration of Helsinki and its latter amendments.

\section{RESULTS}

Five hundred and fifty patients were screened, 82 patients met all the inclusion criteria (Fig. 1). Most of the patients were males $(61 \%)$, with ECOG score $\leq 2(83 \%)$. The median age was 68 years (35-91.5). The main characteristics of these patients are described in Table I. Tumors were peri-hilar, intra-hepatic, distal and gallbladder tumors in respectively 52, 21, 15 and $12 \%$ of patients. Concerning perihilar tumors $(n=43)$, there were Bismuth I, II, III and IV in respectively 4, 14, 14, and 11

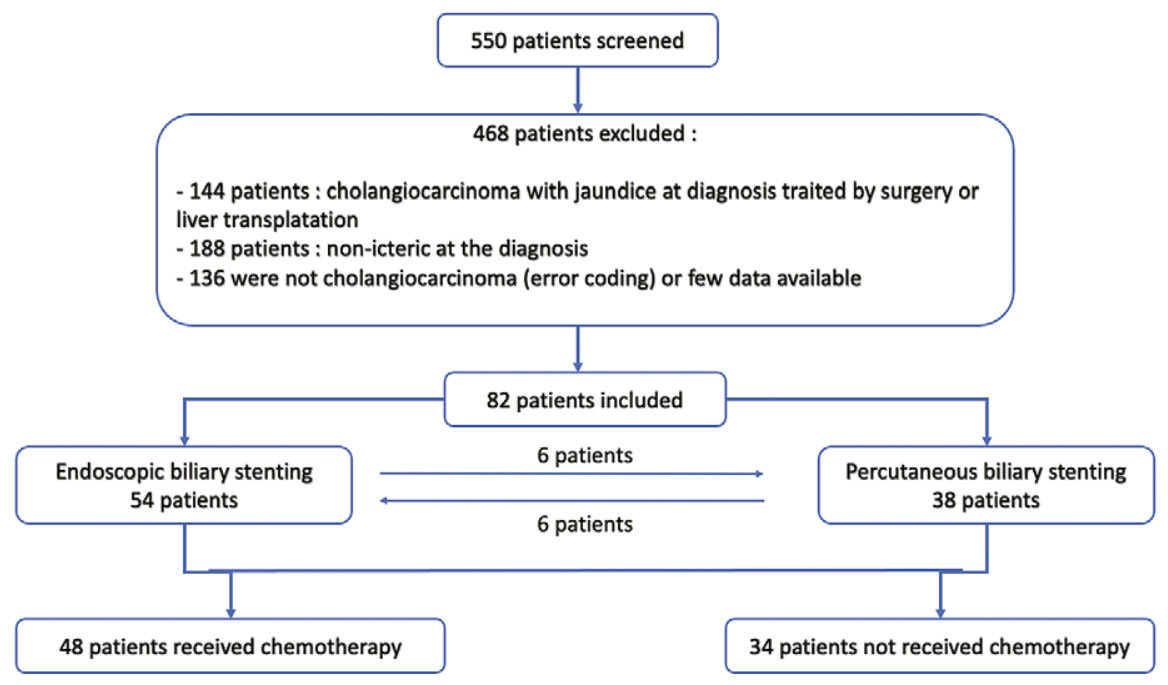

Fig. 1. Flow chart. 
Table I. Baseline patients' characteristics

\begin{tabular}{lcc}
\hline & $\begin{array}{c}\text { Chemotherapy group } \\
\mathrm{N}=48\end{array}$ & $\begin{array}{c}\text { Without chemotherapy } \\
\text { group N=34 }\end{array}$ \\
\hline Age (years), range & $65.8(35-81.5)$ & $73.9(35.2-91.5)$ \\
Male gender, N (\%) & $34(70.83)$ & $16(47.05)$ \\
ECOG score $\leq 2, \mathrm{~N}(\%)$ & $46(95.83)$ & $19(55.88)$ \\
Weight loss (kg) & $7.8(0-26.7)$ & $6.8(0-22.7)$ \\
Localization, N (\%) & & \\
$\quad$ Intrahepatic & $9(18.75)$ & $8(23.52)$ \\
$\quad$ Periilar & $22(45.83)$ & $21(61.76)$ \\
$\quad$ Distal & $11(22.91)$ & $1(2.94)$ \\
$\quad$ Gallblader & $6(12.5)$ & $4(11.76)$ \\
Cholangitis, N (\%) & $4(8.33)$ & $4(11.76)$ \\
Pruritus, N (\%) & $23(47.91)$ & $22(64.7)$ \\
Bilirubin level ( $\mu$ mol/L) & $186.5(61-538)$ & $192(26-589)$ \\
Albumin level, N (\%) & & \\
$\quad \leq 35$ g/L & $15(31.25)$ & $14(41.17)$ \\
$\quad>35$ g/L & $14(29.16)$ & $2(5.88)$ \\
C-reactive protein (mg/L) & $17.9(0-130)$ & $33.9(3-157)$ \\
C-reactive protein/albumin ratio & $0.5(0.05-2.75)$ & $1.5(0.3-7.85)$ \\
Leucocytes counts $(/ \mathrm{mL})$ & $8,200(3,600-14,800)$ & $9,200(4,600-17,200)$ \\
Neutrophils counts $(/ \mathrm{mL})$ & $5,800(1,070-9,580)$ & $6,730(1,049-14,140)$ \\
Time between diagnosis and drainage (days) & $12(0-63)$ & $11.5(0-35)$ \\
Bilirubin level at drainage $(\mu$ mol/L) & $292(98-711)$ & $298.5(62-637)$ \\
Drainage technique, N $(\%)$ & & \\
$\quad$ Percutaneous & $27(32.93)$ & $27(32.93)$ \\
$\quad$ Endoscopic & $21(25.61)$ & $21(25.61)$ \\
Early complication of drainage, N (\%) & $29(60.41)$ & $16(47.05)$ \\
Failure of a previous technique of drainage, N $(\%)$ & $6(12.5)$ & $6(17.64)$ \\
\hline & &
\end{tabular}

patients. The median level of bilirubin at initial diagnosis was $189.5(26-589) \mu \mathrm{mol} / \mathrm{L}$, and $292(62-711) \mu \mathrm{mol} / \mathrm{L}$ at the time of drainage.

Fifty-four patients (66\%) had an endoscopic biliary stenting and twenty-eight (44\%) a percutaneous one. Twelve patients had a technical failure of the initial drainage procedure, requiring a second procedure by the alternative way of drainage with technical success. Median time between diagnosis and biliary drainage was 12 (0-63) days.

Forty-eight patients (59\%) received chemotherapy. Median time between biliary drainage and first chemotherapy cycle was 42 days (6-380). The median bilirubin level for patients at the time of chemotherapy introduction was $27.80(10-444)$ $\mu \mathrm{mol} / \mathrm{L}$. Median duration of chemotherapy was $122( \pm 88.47)$ days. Twenty-five patients (52\%) received gemcitabine plus oxaliplatin as first line chemotherapy, 11 (23\%) gemcitabine plus cisplatin, $4(8.3 \%)$ Folfox (leucovorin calcium (folinic acid), fluorouracil, and oxaliplatin), 3 (6.3\%) gemcitabine alone, 2 (4.1\%) LV5FU2 (fluorouracil and leucovorin) plus cisplatin and $3(6.3 \%)$ a combination of gemcitabine, oxaliplatin and regorafenib. Ten patients (17\%) of patients who received chemotherapy were $\geq 75$ years old.

The main reason for not administrating chemotherapy was notified in the medical records for 27 patients: persistent poor general condition $(n=16)$, patient's refusal $(n=5)$, inadequate persistent jaundice $(n=2)$, recurrent cholangitis $(n=2)$, and 2 patients died early.
We compared the group of patients who received chemotherapy and the group of patients who did not receive chemotherapy (Supplementary Table I). In univariate analysis, young age $(p=0.0014)$, male gender $(p=0.03)$, ECOG score $\leq 2$ at diagnosis $(\mathrm{p}<0.001)$, albumin level at diagnosis $(\mathrm{p}=0.023), \mathrm{CRP}$ level at diagnosis $(p=0.046)$, endoscopic drainage $(p=0.029)$ were significantly associated with chemotherapy introduction. The CAR was calculated, with a significant difference between the two groups $(p=0.017)$.

In the multivariate analysis, ECOG score $\leq 2$ at diagnosis $(\mathrm{HR}=70.5$; 95\%CI: 4.5-1097.6; $\mathrm{p}=0.002)$ and male gender $(\mathrm{OR}=5$; 95\%CI: $1.5-16.5 ; \mathrm{p}=0.009)$ were the only significant independent predictive factors of chemotherapy introduction.

Median OS was 4.9 months (0.2-38.7) in the group of patients who did not receive chemotherapy and 12.2 months (1.9-61.0) in the group of patients who received chemotherapy $(\mathrm{HR}=2.93$; 95\%CI: 1.6-5.3; $\mathrm{p}<0.0001)$ (Fig. 2). Median OS was 12.7 months (1.9-61.0) for patients with bilirubin level $<1.5 \mathrm{x}$ upper limit normal (ULN) at the introduction of chemotherapy, and 10.8 months (2.2-50.2) for patients with bilirubin level $>1.5 x U L N$ at the introduction of chemotherapy $(\mathrm{HR}=0.8$; 95\%CI: 0.5-1.5; $\mathrm{p}=0.57)$.

In univariate analysis, chemotherapy $(\mathrm{p}<0.0001), \mathrm{ECOG}$ score $\leq 2$ at diagnosis $(p=0.0003)$, younger age $(p=0.041)$ and male gender $(p=0.025)$ were significant factors associated with better OS, while gallbladder cancers were associated with a poor OS $(\mathrm{p}=0.034)$. In multivariate analysis, chemotherapy 


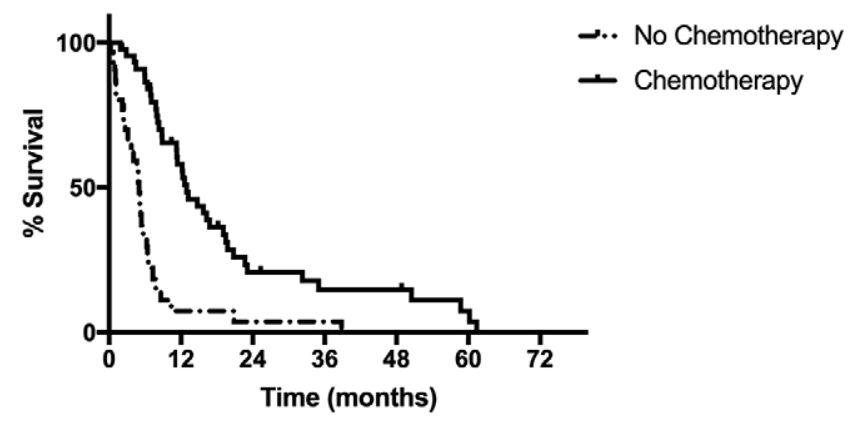

Fig. 2. Survival curves.

(HR=0.33; 95\%CI: 0-0.6; $\mathrm{p}=0.001)$ was associated with better OS while gallbladder tumors had a poor prognostic factor (HR=2.50; 95\%CI: 1.1-5.8; $\mathrm{p}=0.031)$ (Supplementary Table II).

Median PFS was 8.9 months (1.6-32.6). Median PFS was 8.8 months (4.0-32.6) for patients with bilirubin level $<1.5 \mathrm{xULN}$ at the introduction of chemotherapy, and 9 months (1.6-20.8) for patients with bilirubin level $>1.5 x$ ULN at the introduction of chemotherapy (HR=0.64; 95\%CI: 0.3-1.4; $\mathrm{p}=0.38$ ).

Few data were available in the medical records regarding the toxicity of chemotherapy. Twenty-four patients had a reported grade $\leq 3$ hematological or neurological toxicity of chemotherapy. There was no difference between patients with bilirubin level $<1.5 \mathrm{xULN}$ at the introduction of chemotherapy and those with bilirubin level >1.5xULN (95\%CI: 0.4-7.4; $\mathrm{p}=0.74$ ).

\section{DISSCUSION}

To our knowledge, in this clinical setting of patients with advanced BTC requiring biliary drainage, this is the first study investigating the initiation of chemotherapy rate after biliary drainage and the associated factors. In our study, 59\% of patients received chemotherapy after biliary drainage. ECOG score $\leq 2$ and male gender were the only independent predictive factors of initiation of chemotherapy. Median OS of patients treated with chemotherapy was 12.2 months, in range of results from the literature (11 months in the Gemcitabine and Cisplatin combination arm of the ABC-02 trial) [1].

It is important to highlight the factors that were not predictive of the initiation of chemotherapy, notably age and bilirubin level at diagnosis. Older age should not be considered as a formal contraindication of chemotherapy. A geriatric assessment should be proposed in elderly patients, and the management could be discussed in multidisciplinary team, in particular to avoid undertreatment [5]. As an example, the G8 geriatric assessment tool is a quick and easy-to-use screening tool for patient under 70 years old that can be performed routinely by oncologists. It is an appropriate method for identifying older cancer patients that may need a comprehensive geriatric assessment [6].

In the phase III ABC-02 trial investigating gemcitabine plus cisplatin compared to gemcitabine alone, bilirubin level $>1,5$ ULN was an exclusion criteria. In a phase I study, elevated bilirubin level was associated with increased toxicity of gemcitabine [7]. However, another study did not demonstrate increased toxicity of gemcitabine-cisplatin combination in patients treated for advanced BTC [8]. In our study, the median bilirubin level for patients at time of chemotherapy was $30(10-444) \mu \mathrm{mol} / \mathrm{L}$. Almost $50 \%$ of the patients received chemotherapy while the bilirubin level was $>1,5 x U L N$. Overall survival of patients receiving chemotherapy was similar between patients with bilirubin superior or inferior to $1,5 \times$ ULN. It highlights that biological and clinical criteria in daily clinical practice can differ from those in clinical trials, and that normal or subnormal bilirubin level is not mandatory to initiate chemotherapy [8]. The pharmacology of gemcitabine and cisplatin are not known to be significantly impaired in the context of high bilirubin level.

In our study, male gender was a significant factor for chemotherapy introduction, both in univariate and multivariate analysis. In the ABC-02 study and in a large study from the Surveillance, Epidemiology, and End Results Program, male gender was associated with increased mortality $[1,9]$. In our study, we could not identify any explanation to these data.

ECOG score is an independent prognostic factor for OS and PFS in advanced biliary tract cancer, in first or second line of chemotherapy [1,5-7]. ECOG score was also prognostic in univariate analysis in our study but not in multivariate analysis. ECOG was an independent predictive factor for chemotherapy introduction.

The serum level of CRP was also associated with the initiation of chemotherapy in univariate analysis, but cholangitis and leukocyte count at diagnosis were not. In a meta-analysis of 10 studies including 4,592 patients with solids cancers, the CAR was significantly associated with poor overall survival [10]. In our study, the CAR was a significant predictive factor in univariate analysis. However, due to few albumin and CRP levels available in the medical records, this factor could not be included in multivariate analysis.

This study has some limitations. First, this is retrospective study, leading to biases and missing data. Some factors could not be evaluated, such as percentage of drained liver or other biological values including Carbohydrate antigen 19-9 and lactate dehydrogenase. However, this was a homogeneous series of patients with advanced BTC and jaundice, excluding other cancers. In this setting, this is the first study not only focusing on the bilirubin level after biliary drainage, but also investigating the initiation of chemotherapy rate, which may be a more clinically relevant endpoint in daily practice.

\section{CONCLUSIONS}

Our study found that ECOG score $\leq 2$ and male gender were the only independent predictive factors of chemotherapy introduction in BTC in patients with advanced BTC and obstructive jaundice. Age and bilirubin levels were not predictive factors. Further prospective studies should better define predictive factors that will help defining and optimizing the therapeutic strategy in patients with advanced BTC and obstructive jaundice.

Conflicts of interest: None to declare. 
Authors' contributions: M.A. and Y.T. conceived and designed the study. M.A., A. Lièvre, H.S., P.G., J.M., J.B., T.W., M.S., J.G., T.M-B., A. Lim, J.E. collected the data. M.A., B.D., Y.T. analyzed the data. M.A., T.M-B., Y.T. analyzed the data. A. Lièvre, H.S., B.D., P.G., J.M., J.B., T.W., M.S., J.G., T.M-B., A. Lim, J.E. critically revised the manuscript. All the authors approved the final version of the paper.

Supplementary material: To access the supplementary material visit the online version of the J Gastrointestin Liver Dis at http://dx.doi. org/10.15403/jgld-3120.

\section{REFERENCES}

1. Valle J, Wasan H, Palmer DH, et al. Cisplatin plus Gemcitabine versus Gemcitabine for Biliary Tract Cancer. N Engl J Med 2010;362:12731281. doi:10.1056/NEJMoa0908721

2. Nakeeb A, Pitt HA, Sohn TA, et al. Cholangiocarcinoma. A spectrum of intrahepatic, perihilar, and distal tumors. Ann Surg 1996;224:463-473. doi:10.1097/00000658-199610000-00005

3. Monson JR, Donohue JH, McEntee GP, et al. Radical resection for carcinoma of the ampulla of Vater. Arch Surg 1991;126:353-357. doi:10.1001/archsurg.1991.01410270099016
4. Regimbeau JM, Fuks D, Bachellier P, et al. Prognostic value of jaundice in patients with gallbladder cancer by the AFC-GBC-2009 study group. Eur J Surg Oncol 2011;37:505-512. doi:10.1016/j.ejso.2011.03.135

5. Soubeyran P, Bellera C, Goyard J, et al. Screening for Vulnerability in Older Cancer Patients: The ONCODAGE Prospective Multicenter Cohort Study. PLoS One 2014;9:e115060. doi:10.1371/journal.pone.0115060

6. Bellera CA, Rainfray M, Mathoulin-Pélissier S, et al. Screening older cancer patients: first evaluation of the G-8 geriatric screening tool. Ann Oncol 2012;23:2166-2172. doi:10.1093/annonc/mdr587

7. Venook AP, Egorin MJ, Rosner GL, et al. Phase I and pharmacokinetic trial of gemcitabine in patients with hepatic or renal dysfunction: Cancer and Leukemia Group B 9565. J Clin Oncol 2000;18:2780-2787. doi:10.1200/JCO.2000.18.14.2780

8. Lamarca A, Benafif S, Ross P, Bridgewater J, Valle JW. Cisplatin and gemcitabine in patients with advanced biliary tract cancer $(A B C)$ and persistent jaundice despite optimal stenting: Effective intervention in patients with luminal disease. Eur J Cancer 2015;51:1694-1703. doi:10.1016/j.ejca.2015.05.018

9. Bridgewater J, Lopes A, Wasan H, et al. Prognostic factors for progression-free and overall survival in advanced biliary tract cancer. Ann Oncol 2016;27:134-140. doi:10.1093/annonc/mdv483

10. Li N, Tian GW, Wang Y, Zhang H, Wang ZH, Li G. Prognostic Role of the Pretreatment C-Reactive Protein/Albumin Ratio in Solid Cancers: A Meta-Analysis. Sci Rep 2017;7:41298. doi:10.1038/srep41298 